\title{
In Vitro Performance Testing of Medicated Chewing
}

\section{Gums}

Lisa Zieschang ${ }^{1,2, *}$, Martin Klein ${ }^{1}$, Johannes Krämer ${ }^{1}$, and Maike Windbergs ${ }^{2,3}$

${ }^{1}$ Eurofins - PHAST Development, GmbH \& Co. KG, Konstanz, Germany

${ }^{2}$ Institute of Pharmaceutical Technology and Buchmann Institute for Molecular Life Sciences, Goethe-University Frankfurt, Frankfurt, Germany

${ }^{3}$ PharmBioTec GmbH, Saarbruecken, Germany

\section{ABSTRACT}

e-mail: windbergs@em.uni-frankfurt.de

The oromucosal route of therapeutic administration can be targeted by medicated chewing gums (MCGs) loaded with locally or systemically acting active pharmaceutical ingredients (API). Compared to other dosage forms, release of the API is mainly determined and controlled by the patient's mastication. These unique characteristics create attractive opportunities for the delivery of API, but at the same time, they challenge safety and efficacy of the drug product. In vitro release testing is a vital tool for the assessment of the drug product's quality and performance, but the pharmacopeial procedure is solely described in the European Pharmacopoeia. Given this background, this review focuses on parameters influencing in vitro release testing of MCG related to the testing device, the formulation and manufacturing of MCG, as well as the operational parameters adapted to the physiological conditions in the oral cavity. Furthermore, the unique features of MCG for a reliable in vitro-in vivo correlation approach will be introduced along with the existing hurdles to standardise and verifiy the methods for MCG release testing.

KEYWORDS: Medicated chewing gum, in vitro performance testing, IVIVC; drug delivery, dissolution

\section{INTRODUCTION}

I ven though chewing gums are commonly used confectionary products since decades, their application as drug delivery systems currently provokes increasing interest. Besides encapsulation of diagnostics, medicated chewing gums (MCGs) for therapeutic use can be loaded with a wide range of different active pharmaceutical ingredients (API) intended for local action in the oral cavity or for systemic action after absorption through the oral mucosa and/ or the gastrointestinal $(G I)$ tract $(1,2)$. The European Pharmacopoeia (Ph. Eur.) specifies MCG as "solid, singledose preparations with a base consisting mainly of gum, that are intended to be chewed but not swallowed". MCG are defined in the United States Pharmacopeia (USP), Ph. Eur., and Japanese Pharmacopeia (JP), emphasizing their application as drug delivery system (2-4). Compared to other solid dosage forms, the release of API from MCG is mainly triggered by the patient while chewing, and there is the opportunity to terminate the delivery by removing the MCG from the oral cavity. The mastication process is mainly needed to create new surfaces for the release of the drug substance. Unlike for classical solid oral dosage forms, such as tablets with a spontaneous dissolution process, masticatory activities are a prerequisite for continuous drug release from chewing gums (5).

In addition, the manufacturing processes (melting/ extrusion, direct compression) differ from those applied for other solid dosage forms $(4,6)$. The resulting chewing gums manufactured by direct compression are also referred to as chewing gum tablets due to the production process, and the gum-forming behaviour is highly affected by the drug loading $(7,8)$. In the literature, a clear differentiation is needed for chewing gum, chewable tablets, and chewable gels. Unlike chewing gums, chewable tablets are intended to be swallowed after chewing or crushing, which also applies to chewable gels and chewable soft gel capsules $(4,9)$.

MCG may be used for loading with locally or systemically acting API (2). Their absorption throughout the oral mucosa provides direct access to the systemic blood circulation while avoiding the hepatic first-pass effect. Saliva acts as the physiological dissolution medium in the oral cavity once the drug substance is released from the MCG. It mainly consists of water (99.5\%), a comparatively small percentage of proteins, acting as surfactants or digestive enzymes, as well as sodium,

*Corresponding author 
chloride, and bicarbonate (10). Saliva composition and $\mathrm{pH}$, as well as the secreted volume, underlie high intraand inter-individual variability and depend on several factors, like health of the patient, age, and sex, as well as the palatability of the chewing gum (11-13).

With focus on the physiological conditions of the oral cavity and mastication behaviour in vivo, in vitro performance tests for MCG should be designed to investigate the release of API from chewing gums under reproducible conditions. Two compendial devices for in vitro release testing of MCG are described in Ph. Eur., which can be used either for quality control or drug product development $(5,14)$. The USP does not provide a general chapter about chewing gums, but includes a monograph for nicotine polacrilex gum without in vitro performance testing protocols (15). Product performance tests for MCG are briefly mentioned in the general chapter for mucosal drug products-performance tests of the USP, whereas for additional information (e.g., the usage of devices), the reader is referred to corresponding chapters in the Ph. Eur. (16). Furthermore, no additional information about release testing of MCG is provided by the JP.

This review article intends to provide information about the state of the art performance testing of MCG with emphasis on factors influencing the release kinetics, in vitro and in vivo, as well as their correlation.

\section{REGULATORY REQUIREMENTS FOR MCG}

The Ph. Eur. denotes the performance test for MCG, "Dissolution test for medicated chewing gums," although no dissolving of the drug product takes place. Since the delivery system remains intact and it releases the API, this article will use the term "release test" to describe the performance test for MCG (17).

Quality tests for MCG are generally related to the regulatory requirements for solid, oral dosage forms (2, 15). Since the MCG needs to be activated by mechanical forces in an aqueous environment at body temperature to release the API, the testing device needs to mimic the physiological mastication process. Within the area harmonized by $\mathrm{ICH}, \mathrm{Ph}$. Eur. is the only pharmacopeia describing two different instruments, both as closed systems (Fig. 1). Apparatus A, as described in the Ph. Eur., consists of a non-transparent metal chamber, two horizontal oscillatory testing device pistons, which simulate the mastication, and one vertical piston to keep the chewing gum in place during release testing. Apparatus B, as described in the Ph. Eur., consists of a double walled glass chamber, including one vertical oscillatory piston and one stationary rotating piston with removable chewing jaws (14). The jaws of apparatus $B$ need to be replaced and qualified for their thickness and surface roughness after each run, to ensure reproducible results $(5,14)$. The Ph. Eur. recommends to operate both devices using $20 \mathrm{~mL}$ of phosphate buffer $\mathrm{pH} 6.0$ at $37^{\circ} \mathrm{C} \pm$ $0.5^{\circ} \mathrm{C}$ (14). Additionally, according to the manufacturer's
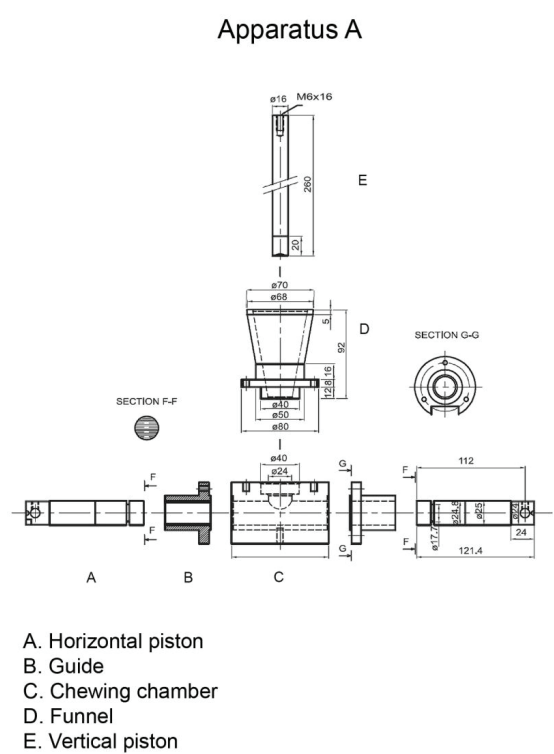

A. Horizontal piston

B. Guide

C. Chewing
D. Funnel

E. Vertical piston
Apparatus B

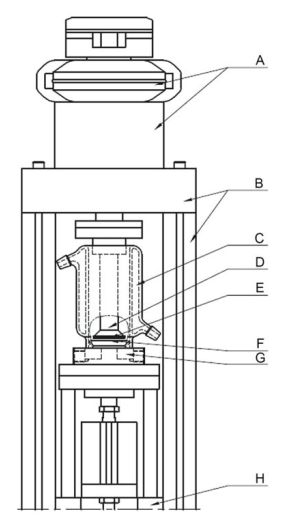

$$
\begin{array}{ll}
\text { A. Revolving device for the } & \text { E. Upper chewing surface } \\
\text { upper chewing surface } & \text { F. Lower chewing surface } \\
\text { B. Stand } & \text { G. Base chamber } \\
\text { C. Test cell } & \text { H. Device for up-and-down } \\
\text { D. Axle } & \text { chewing motion }
\end{array}
$$

Figure 1. Schematic representation of apparatus $A$ and $B$, modified with permission from Ph. Eur. (14). 
information for apparatus $B$, it is possible to increase the volume up to $70 \mathrm{~mL}$, whereas it is not known if this possibility exists for apparatus $A$. Unlike apparatus $A$, apparatus $\mathrm{B}$ is commercially available in a compact, modular design (18).

Release testing of MCG is usually carried out in six runs $(n=6)$, with a chewing frequency of 60 strokes per minute. It is suggested to operate a multi-point release test or determine the content of the remaining API in the chewed gum to obtain a drug release profile as a function of time with the amount of released API expressed as a percentage of the label claim $(14,17)$.

\section{IN VITRO PERFORMANCE TESTING OF MCG Physiological Factors Affecting Drug Release}

The masticatory process is based on a complex physiological mechanism and comprises the principal part of the release of API from MCG in vivo, and thus must be simulated for predictive in vitro testing. Before mastication, the administered chewing gum is solid and shows hardly any release upon addition of dissolution medium. After formation of the gum bolus by chewing, the MCG is activated and the API is released under mastication. Mechanical forces, temperature, wettability, and water permeation rate are factors influencing the transformation of the dosage form from a solid to semisolid state. By kneading the gum with the teeth, new surfaces for drug release are created due to the plasticity of the activated gum and the mechanical force applied during each chewing operation. The chewing frequency is a key parameter that determines the time needed for the maximum release, whereas the total release of the API depends on the number of strokes (5).

The dissolution medium in vivo is saliva, which is classified into stimulated and unstimulated saliva depending on the flow rate (production) and composition (11). Several investigations show a variable $\mathrm{pH}$ range of unstimulated and stimulated saliva from 6.1 to $7.7(11,19-21)$. Stimulated saliva is characterized by an increased concentration of phosphate-, protein- and especially bicarbonatebuffer systems, which are responsible for higher $\mathrm{pH}$ values, compared to unstimulated saliva. For instance, Gittings et al examined unstimulated and stimulated saliva separately and found $\mathrm{pH}$ values of 6.5-7.3 for unstimulated and 7.0-7.7 for stimulated saliva, which led them to the conclusion that dissolution media should also be categorized to reflect the physiological situation in the oral cavity (11). The buffer capacity of saliva also shows a high inter-individual variation in vivo and similar to the $\mathrm{pH}$, the buffer capacity is higher for stimulated saliva
(11). The saliva flow rate was found to be in the range of $0.05-3.45 \mathrm{~mL} / \mathrm{min}$ in humans, regardless of whether the flow rate of unstimulated or stimulated saliva was investigated $(11,19,22)$. High variabilities in the flow rate are due to salivary stimulation affected by mechanical, gustatory, visual, and olfactory mechanisms. Therefore, mouthfeel and taste are important parameters for the patient compliance, and hence the release, since it can affect the salivary flow rate, depending on the flavouring or sweetening agents used (23). The chewing frequency is also a parameter with high inter-individual variety; however, MCG chewed at different frequencies showed no change in the salivary flow rate $(24,25)$.

\section{Parameters of In Vitro Testing Devices Affecting Drug Release}

The parameters of in vitro testing devices - chewing frequency, twisting angle of the jaws, and jaw distance - can be modified while the temperature is usually kept at $37{ }^{\circ} \mathrm{C} \pm 0.5^{\circ} \mathrm{C}$ (14). A general recommendation for adjustments of the release test devices is given by Gajendran et al (26). In principle, faster drug release was observed when the frequency increased from 40 to 60 strokes per minute. The amount of drug released is a function of the number of strokes. Also, an increased twisting angle from 20 to 40 degrees for apparatus B leads to higher drug release. For the jaw distance, a higher release was revealed with decreasing distance for both devices in the following order; apparatus $A$ : $0.7<0.5$ $<0.3 \mathrm{~mm}$; apparatus B: $1.8<1.6<1.4 \mathrm{~mm}$ (Fig. 2) (26). In the case of apparatus $B$, a high chewing frequency in combination with high twisting angles cannot be used, due to a limited rotation speed of the upper piston (27).

For the development and validation of in vitro drug release testing methods for MCG, the same procedures used for solid oral dosage forms described in the USP General Chapter <1092> can be applied in a modified form (28). Additional information about the composition and properties of simulated saliva as biorelevant medium has been provided by Marques et al (29).

The two compendial devices have been included in a collaborative study to test the precision of drug release results for three different types of nicotine chewing gum products in six different laboratories (30). One of the major findings was that there is no general preference for one apparatus and that the devices are not providing similar results for a particular product. Gajendran et al. compared both devices and concluded that for the selected drug products, no discriminatory drug release profiles under different test conditions could be generated when using apparatus $A$, whereas with apparatus $B$, different device 
setups can be reflected in the release profiles in all cases (26).
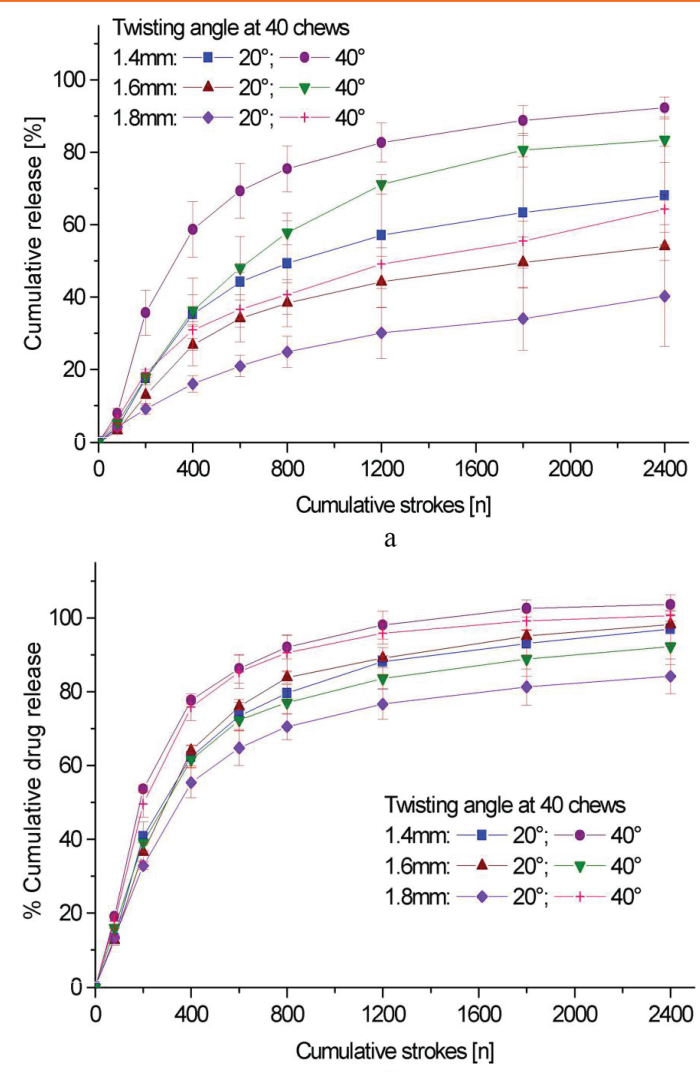

b

Figure 2. Representative in vitro release profiles of two different types of nicotine chewing gums (product $a$ and $b$ ) from studies with apparatus $B$, with different parameter settings.

Data represent mean \pm standard deviation, $n=3$. Reprinted with permission from Wiley and Sons (26).

\section{Effects of Formulation and Manufacturing on Drug Release}

The chewing gum base, as a main ingredient in MCG, is a complex mixture of hydrophobic polymers, which is mainly responsible for the unique properties of the gum in the activated state $(4,6)$. However, depending on the manufacturing method, the release kinetics of the API from MCG may be altered (31). Furthermore, an influence of excipients has been observed for MCG, especially with aromatic compounds, because they play also a role in patient compliance $(6,32-34)$. Additionally, the release of API from the tested gum is increased for hydrophilic compounds and decreased for hydrophobic substances due to solubility in the aqueous medium and the interaction of hydrophobic compounds with the gum matrix (35). For orally disintegrating tablets, suspensions, and gels, size limitations for particles within the delivery systems have been defined for ensuring a pleasant mouthfeel $(36,37)$. In contrast, for chewing gums, the literature is limited to data for sucrose particles in confectionary products, for which a size of $<74 \mu \mathrm{m}$ was shown to be preferential (38). Thus, for each chewing gum formulation, the tolerable size for particulate components must be determined individually (39).

\section{IVIVC}

The USP defines in vitro-in vivo correlation (IVIVC) as "establishment of a rational relationship between a biological property, or a parameter derived from drug plasma concentrations produced by a dosage form, and a physicochemical property or characteristic of the same dosage form" (40). The US Food and Drug Administration (FDA) defines IVIVC as a "predictive mathematical model describing the relationship between an in vitro property of an extended release dosage form (usually the rate or extent of drug dissolution or release) and a relevant in vivo response, e.g., plasma drug concentration or amount of drug absorbed." In the regulatory environment, three levels of correlation are defined depending on the extent of data reduction. Level $A$ is superior and uses the full in vitro and in vivo profiling data (41). Level A correlation of API release from MCG can be achieved without using the general deconvolution approach of the release profiles from plasma concentration profiles after peroral application (Fig. 3) (26). In contrast to disintegrating oral dosage forms, which are no longer accessible after application and decompose in the human body, MCG can be removed from the oral cavity after defined time intervals. Subsequently, the residual API content in MCG can be determined, and thus the released portion of the API can be calculated. With this in vivo information, predictable in vitro tests can be developed, even though the released drug amount does not give any information about the absorption in the human body. Using this approach, the IVIVC of nicotine release from chewing gums was found to be more predictive and accurate compared to the deconvolution method (5). However, as masticatory frequency is a crucial parameter for the drug release, the masticatory frequency needs to be defined in the study design and should be monitored $(26,42)$.

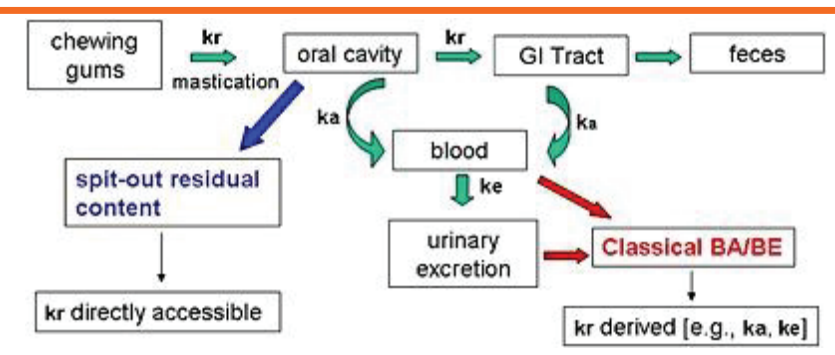

Figure 3. Schematic representation of the in vivo chew-out model compared to the classical BA approach.

$K r$, release rate constant; $G I$, gastrointestinal; ka, absorption rate constant; $k e$, elimination rate constant; $B E$, bioequivalence; $B A$, bioavailability. Reprinted with permission from Wiley and Sons (26). 


\section{CONCLUSION AND OUTLOOK}

Two compendial devices for performance testing of MCG are described in Ph. Eur., but no such methods exist in the USP (14). A collaborative study, however, showed high variability between laboratories and the urgent need for a performance verification test, because the devices need to be handled with care to obtain valid data (30). This may explain why compendial methods compared to Ph. Eur. do not exist in the USP. Comparative studies with release testing devices in different laboratories using a harmonised mechanical qualification procedure, followed by a performance verification test with a reference standard, should be conducted. Furthermore, the influence of device, operator, and formulation on the observed variability of release data should be carefully dissected to exclude the posibility of over- or underdiscrimination of effects on release.

\section{ACKNOWLEDGEMENTS}

The authors disclosed no funding related to this article.

\section{CONFLICT OF INTEREST}

The authors disclosed no conflicts of interest related to this article.

\section{REFERENCES}

1. Ritzer, J.; Lühmann, T.; Rode, C.; Pein-Hackelbusch, M.; Immohr, I.; Schedler, U.; Thiele, T.; Stübinger, S.; Rechenberg, B. V.; Waser-Althaus, J.; Schlottig, F.; Merli, M.; Dawe, H.; Karpíšek, M.; Wyrwa, R.; Schnabelrauch, M.; Meinel, L. Diagnosing peri-implant disease using the tongue as a $24 / 7$ detector. Nat Commun. 2017, 8, 264. DOI: 10.1038/s41467-017-00340-x.

2. Chewing Gums, Medicated. European Pharmacopoeia; European Directorate for the Quality of Medicines, Council of Europe, 9th ed: Strasbourg, FR, 2016; pp 855.

3. General rules for preparations, Preparations for Oro-mucosal Application. The Japanese Pharmacopoeia, English version; Ministry of Health, Labour and Welfare, 17th ed, 2016; pp 12.

4. <1151> Pharmaceutical Dosage Forms. In United States Pharmacopeia and National Formulary USP 41-NF 36; The United States Pharmacopeial Convention, Inc.: Rockville, MD, 2018, pp 1543-1568.

5. Gajendran, J. Performance of medicated chewing gums with the goal of establishing in vitro in vivo correlation. PhD Thesis. Johannes Gutenberg-University, Mainz, Germany. 2016, p 268.

6. Belmar, J.; Ribé, M. Eye on excipients: Direct compression of medicated chewing gum. Tablets Capsules [Online] 2013, 1-4. http://www.healthingum.com/pdf/Eye\%20on\%20excipients. pdf (accessed July 18, 2018).

7. Nissen, V.; Schmidt, N. R.; Andersen, R. B. Compressed chewing gum tablet. European Patent EP 1589825 B9, Feb
4, 2003. European Patent Office Website. https://data.epo. org/publication-server/rest/v1.1/patents/EP1589825W1B1/ document.pdf (accessed July 18, 2018).

8. Al Hagbani, T.; Nazzal, S. Development of postcompressional textural tests to evaluate the mechanical properties of medicated chewing gum tablets with high drug loadings. J. Texture Stud. 2018, 49, 30-37. DOI: 10.1111/jtxs.12287.

9. Dixit, A. S.; Parthasarathi, K. K.; Hosakote, G. S. Gels and jellies as a dosage form for dysphagia patients: a review. Curr. Drug Ther.. 2011, 6, 79-86. DOI: 10.2174/157488511795304921.

10. Squier, C.; Brogden, K.; O'Rourke, J. Human Oral Mucosa Development, Structure and Function; Wiley: Hoboken, NJ, 2011.

11. Gittings, S.; Turnbull, N.; Henry, B.; Roberts, C. J.; Gershkovich, P. Characterisation of human saliva as a platform for oral dissolution medium development. Eur. J. Pharm. Biopharm. 2015, 91, 16-24. DOI: 10.1016/j.ejpb.2015.01.007.

12. Nogourani, M. K.; Janghorbani, M.; Isfahan, R. K.; Beheshti, M. H. Effects of chewing different flavored gums on salivary flow rate and pH. Int. J. Dent. 2012, 569327. DOI: 10.1155/2012/569327.

13. Dawes, C.; Macpherson, L. M. D. Effects of nine different chewing-gums and lozenges on salivary flow rate and $\mathrm{pH}$. Caries Res. 1992, 26, 176-182. DOI: 10.1159/000261439.

14. Dissolution Test for Medicated Chewing Gums Monograph 2.9.25. In European Pharmacopoeia, 9th ed.; European Directorate for the Quality of Medicines, Council of Europe: Strasbourg, France, 2016, pp 340-344.

15. Nicotine Polacrilex Gum Monographs. United States Pharmacopeia and National Formulary USP 41-NF 36; The United States Pharmacopeial Convention, Inc.: Rockville, MD, 2018, pp 5350.

16. $<1004>$ Mucosal Drug Products-Performance Tests. In United States Pharmacopeia and National Formulary USP 41-NF 36; The United States Pharmacopeial Convention, Inc.: Rockville, MD, 2018, pp 6699-6701.

17. Brown, C. K.; Friedel, H. D.; Barker, A. R.; Buhse, L. F.; Keitel, S.; Cecil, T. L.; Kraemer, J.; Morris, J. M.; Reppas, C.; Stickelmeyer, M. P.; Yomota, C.; Shah, V. P. FIP/AAPS joint workshop report: dissolution/in vitro release testing of novel/special dosage forms. AAPS PharmSciTech 2011, 12, 782-794. DOI: 10.1208/ s12249-011-9634-x.

18. Erweka GmbH. Chewing gum tester DRT, http://www.erweka. com/products/dissolution-testers/chewing-gum-tester.html. Accessed June 4, 2018.

19. Aframian, D. J.; Davidowitz, T.; Benoliel, R. The distribution of oral mucosal pH values in healthy saliva secretors. Oral Dis. 2006, 12, 420-423. DOI: 10.1111/j.1601-0825.2005.01217.x.

20. Christersson, C. E.; Lindh, L.; Arnebrant, T. Film-forming properties and viscosities of saliva substitutes and human whole saliva. Eur. J. Oral Sci. 2000, 108, 418-425. DOI: 10.1034/j.16000722.2000.108005418.x.

21. Kazakov, V. N.; Udod, A. A.; Zinkovych, I. I.; Fainerman, V. B.; Miller, R. Dynamic surface tension of saliva: general relationships 
and application in medical diagnostics. Colloid Surface. 2009, 74, 457-461. DOI: 10.1016/j.colsurfb.2009.06.010.

22. Rantonen, P. Salivary flow and composition in healthy and diseased adults. PhD Thesis. University of Helsinki, Helsinki, Finland. 2003, p 99.

23. Humphrey, S. P.; Williamson, R. T. A review of saliva: normal composition, flow, and function. J. Prosthet. Dent. 2001, 85, 162-169. DOI: 10.1067/mpr.2001.113778.

24. Po, J. M. C.; Kieser, J. A.; Gallo, L. M.; Tésenyi, A. J.; Herbison, P.; Farella, M. Time-frequency analysis of chewing activity in the natural environment. J. Dent. Res. 2011, 90, 1206-1210. DOI: 10.1177/0022034511416669.

25. Dong, C.; Puckett, A. D.; Dawes, C. The effects of chewing frequency and duration of gum chewing on salivary flow rate and sucrose concentration. Arch. Oral Biol. 1995, 40, 585-588. DOI: 10.1016/0003-9969(95)00012-E.

26. Gajendran, J.; Kraemer, J.; Langguth, P. In vivo predictive release methods for medicated chewing gums. Biopharm. Drug Dispos. 2012, 33, 417-424. DOI: 10.1002/bdd.1796.

27. Kvist, C.; Andersson, S. B.; Fors, S.; Wennergren, B.; Berglund, J. Apparatus for studying in vitro drug release from medicated chewing gums. Int. J. Pharm. 1999, 189, 57-65. DOI: 10.1016/ S0378-5173(99)00236-7.

28. $<1092>$ The Dissolution Procedure: Development and Validation. In United States Pharmacopeia and National Formulary USP 41-NF 36; The United States Pharmacopeial Convention, Inc.: Rockville, MD, 2018, pp 7178-7198.

29. Marques, M. R. C.; Loebenberg, R.; Almukainzi, M. Simulated biological fluids with possible application in dissolution testing. Dissolution Technol. 2011, 18, 15-28. DOI: 10.14227/ DT180311P15.

30. Brown, W.; Gajendran, J.; Hauck, W.; Krämer, J. Stimuli to the revision process: Collaborative study of the variability of drug release results from nicotine gums using two apparatus designs. USP Pharmacopeial Forum Online website, 2015. www.usppf. com (accessed May 15, 2018).

31. Morjaria, Y.; Irwin, W. J.; Barnett, P. X.; Chan, R. S.; Conway, B. $R$. In vitro release of nicotine from chewing gum formulations. Dissolution Technol. 2004, 11, 12-15. DOI: 10.14227/ DT110204P12.

32. Raithore, $\mathrm{S}$. Effect of polyols on flavor release during mastication of sugar-free confections. PhD Thesis. University of Minnesota, Minneapolis, MN, USA. 2012, p 166.
33. Akbal, O.; Cevher, E.; Araman, A. O. The development and in vitro evaluation of benzydamine hydrochloride medicated chewing gum formulations. Istanbul J. Pharm. 2017, 47, 45-51. DOI: 10.5152/IstanbulJPharm.2017.007.

34. Al-Ghananeem, A. M.; Leung, K. P.; Faraj, J.; DeLuca, P. P. Development of a sustained antiplaque and antimicrobial chewing gum of a decapeptide. AAPS PharmSciTech 2017, 18, 2240-2247. DOI: 10.1208/s12249-016-0706-9.

35. Rassing, M. R. Chewing gum as drug delivery system. Adv. Drug. Deliver. Rev. 1994, 13, 89-121. DOI: 10.1016/0169409X(94)90028-0.

36. Kimura, S.-I.; Uchida, S.; Kanada, K.; Namiki, N. Effect of granule properties on rough mouth feel and palatability of orally disintegrating tablets. Int. J. Pharm. 2015, 484, 156-162. DOI: 10.1016/j.ijpharm.2015.02.023.

37. Imai, E.; Hatae, K.; Shimada, A. Oral perception of grittiness: effect of particle size and concentration of the dispersed particles and the dispersion medium. J. Texture Stud. 1995, 26, 561-576. DOI: 10.1111/j.1745-4603.1995.tb00804.x.

38. Hartel, R. W.; von Elbe, J. H.; Hofberger, R. Confectionery Science and Technology; Springer International Publishing AG: Cham, DE, 2017; pp 536.

39. Shabir, Q.; Skaria, C.; Brien, H. O.; Loni, A.; Barnett, C.; Canham, L. Taste and mouthfeel assessment of porous and non-porous silicon microparticles. Nanoscale Res. Lett. 2012, 7, 407. DOI: 10.1186/1556-276X-7-407.

40. <1088> In Vitro and In Vivo Evaluation of Dosage Forms. In United States Pharmacopeia and National Formulary USP 41-NF 36; The United States Pharmacopeial Convention, Inc.: Rockville, MD, 2018, pp 7159-7170.

41. Guidance for Industry: Extended Release Oral Dosage Forms: Development, Evaluation, and Application of In Vitro/In Vivo Correlations; U.S. Department of Health and Human Services, Food and Drug Administration, Center for Drug Evaluation and Research (CDER): Washington, US, 1997; pp 1-24.

42. Kvist, L. C.; Andersson, S.-B.; Berglund, J.; Wennergren, B.; Fors, S. M. Equipment for drug release testing of medicated chewing gums. J. Pharm. Biomed. 2000, 22, 405-411. DOI: 10.1016/ S0731-7085(99)00307-6. 